\title{
A long term study of pulmonary function among US refractory ceramic fibre workers
}

\author{
Roy T McKay, ${ }^{1}$ Grace K LeMasters, ${ }^{1}$ Timothy J Hilbert, ${ }^{1}$ Linda S Levin, ${ }^{1}$ Carol H Rice, ${ }^{1}$ \\ Eric K Borton, ${ }^{1}$ James E Lockey ${ }^{1,2}$
}

${ }^{1}$ Department of Environmental Health, University of Cincinnati College of Medicine, Cincinnati, Ohio, USA

${ }^{2}$ Department of Internal

Medicine (Pulmonary Division), University of Cincinnati College of Medicine, Cincinnati, Ohio, USA

\section{Correspondence to}

Roy T McKay, Department of Environmental Health, ML 0056, University of Cincinnati College of Medicine, Cincinnati, $\mathrm{OH}$ 45267-0056, USA; roy.mckay@uc.edu

Accepted 6 January 2010 Published Online First 25 August 2010

\section{ABSTRACT}

Background Cross-sectional studies have shown declines in lung function among refractory ceramic fibre (RCF) workers with increasing fibre exposure. This study followed current and former workers ( $n=1396)$ for up to 17 years and collected 5243 pulmonary function tests. Methods Cumulative fibre exposure and production years were categorised into exposure levels at five manufacturing locations. Conventional longitudinal models did not adequately partition age-related changes from other time-dependent variables. Therefore, a restricted cubic spline model was developed to account for the non-linear decline with age.

Results Cumulative fibre $>60$ fibre-months/cc showed a significant loss in lung function at the first test. When results were examined longitudinally, cumulative exposure was confounded with age as workers with the highest cumulative exposure were generally older. A longitudinal model adjusted by age groups was implemented to control for this confounding. No consistent longitudinal loss in lung function was observed with RCF exposure. Smoking, initial weight and weight increase were significant factors.

Conclusion No consistent decline was observed longitudinally with exposure to RCF, although crosssectional and longitudinal findings were discordant. Confounding and accelerated lung function declines with ageing and the correlation of multiple time-dependent variables should be considered in order to minimise error and maximise precision. An innovative statistical methodology for these types of data is described.

\section{INTRODUCTION}

Refractory ceramic fibres (RCF) are man-made vitreous fibres used for high temperature insulation applications. Final products may consist of bulk fibre, blankets, boards, paper and textiles. Health concerns regarding RCF exposure are related to fibres that are of respirable size and relatively persistent in the lungs. Initial RCF inhalation studies in rats and hamsters found interstitial and pleural fibrosis, lung cancer and mesothelioma at maximum tolerated doses. Additional inhalation studies using lower levels of exposure demonstrated a cellular response at $36 \mathrm{f} / \mathrm{cc}$, a single mesothelioma at $91 \mathrm{f} / \mathrm{cc}$, and minimal fibrosis at 91 and $162 \mathrm{f} /$ cc. $^{1-4}$ A cross-sectional evaluation of workers in seven European plants that manufactured RCF showed a decrease in $\mathrm{FEV}_{1}$ with increasing cumulative fibre exposure in current and former smokers. ${ }^{5}$ Our previous cross-sectional analysis demonstrated a significant decrease in FVC for 552 RCF male workers after adjusting for smoking. ${ }^{6}$

\section{What this paper adds}

- Cross-sectional studies have shown declines in lung function among refractory ceramic fibre (RCF) workers with increasing fibre exposure.

- No longitudinal studies of this length have been conducted on RCF exposed workers and little is known about female and former workers.

- This study showed no consistent exposurerelated decline in lung function among currently exposed men and women or former male workers.

- Cumulative fibre exposure was found to be highly correlated with other time-dependent variables.

- Traditional longitudinal models may not account for non-linear, age-related declines in lung function.

We previously reported no significant decline in lung function in a longitudinal analysis among 361 male current workers providing pulmonary function tests (PFT) over 7 years. ${ }^{6}$ The primary purpose of this study is to expand the previous report by evaluating 1396 participants including females and former workers followed for up to 17 years. This extended longitudinal study period, however, posed analytical challenges regarding the partitioning of age-related changes from other time-dependent exposure variables.

\section{METHODS \\ Subjects}

Spirometry tests were offered to current male and female workers at five RCF manufacturing locations. At four of these locations, tests were performed yearly from 1987 to 1994 and then every 3 years through 2004. At the fifth location, tests were conducted yearly from 1987 to 1997, when testing was terminated. Those who left employment prior to 1987, that is, former workers, were evaluated every 3 years at two locations.

A total of 1290 male and female current workers were eligible, having 1 or more years of RCF employment at study initiation or 1 month prior to testing after study initiation. Of these, 75 salesmen employed elsewhere were excluded. Thirty workers having current asthma for over half of their test dates were removed because asthma medications could introduce an uncharacterised effect on PFT results. Of the 1185 workers remaining, 99.3\% $(1177 / 1185)$ provided at least one PFT. Eight did not 
provide a PFT. There were 933 eligible males (67.7\% Caucasian, $27.7 \%$ African American and 4.6\% other) and 244 females (89.3\% Caucasian, 9.4\% African American and 1.2\% other). Their overall testing rate was $94.2 \%$ (5243 tests/5566 opportunities). Former workers within a 50-mile radius of two facilities were also invited to participate $(n=272)$. Of these, 219 males (too few females) participated who did not have current asthma symptoms and were not in sales. Former workers who moved out of state were initially scheduled for PFTs at local clinics; however, test quality was so poor the results were unusable. Additional details regarding subject recruitment are provided elsewhere. ${ }^{6}$

\section{Exposure estimation}

Evaluation of current exposures and reconstruction of historical exposures have been described. ${ }^{7}$ In 1987, a standardised protocol was implemented for full-shift quarterly sampling of randomly selected workers. Historical exposures were reconstructed for two manufacturing locations where measurements and operating records were available. To provide exposure estimates for jobs never sampled, similarities in operations were used to impute a value. For this report, estimates of exposure prior to 1987 were developed for three manufacturing locations not previously reported. These estimates utilised previous categorisations of jobs into industry-wide job title groups or titles involving similar activities and were reviewed by plant personnel. The assumptions were that across industries, technology changed at generally the same pace and the ratio of pre- and post-1987 exposures did not vary appreciably, although the magnitude may have differed. For example, if the 1987 exposure at company A was $0.45 \mathrm{f} / \mathrm{cc}$ where no pre-1987 value was available, the exposure for a similar job activity was compared with the 1987 values for all other locations where prior exposures had been available or reconstructed. Assume the closest 1987 job activity value at company B was $0.40 \mathrm{f} / \mathrm{cc}$, but its pre-1987 exposure value was $0.80 \mathrm{f} / \mathrm{cc}$. Then the ratios of 1987 to pre-1987 exposures were used to calculate the unknown $\mathrm{x}$, as $((0.40 / 0.80)=(0.45 / \mathrm{x}))$, allowing assignment of $0.90 \mathrm{f} / \mathrm{cc}$ for the unmeasured time period at company A. These methods increased the number of subjects with calculated lifetime cumulative fibre exposure by 282 .

\section{Spirometry}

Spirometry testing was conducted by experienced University of Cincinnati technicians who had completed a National Institute for Occupational Safety and Health approved training course. ${ }^{6}$ At study initiation, testing was conducted with Ohio-Med 822 dry rolling seal spirometers (Ohio Medical Products, Madison, Wisconsin, USA). In 1998 the spirometers were changed to a similar style (SensorMedics 922 with OMI software; SensorMedics, Houston, Texas, USA). Calibration checks and quality control procedures met and exceeded those recommended by standard setting organisations and the spirometer manufacturer. During PFT visits, volume calibration checks were performed several times a day in order to evaluate measurement error. The average volume difference for the five spirometers was very small and ranged from -38 to $+44 \mathrm{ml}$. When a study participant provided a PFT, the spirometer ID was recorded, and the measured volume was adjusted according to the correction factor for each spirometer.

\section{Statistical methods}

Cross-sectional and longitudinal analyses were performed to examine the associations of spirometry values and RCF exposure. The primary analyses were for 933 current male employees having a PFT between 1987 and 2004. FVC and $\mathrm{FEV}_{1}$ values were corrected for body size by dividing by the square of the subject's height at time of test. ${ }^{6}$ For ease of interpretation, the corrected values were then converted to volume units by multiplying by the square of mean height. Predicted values used for comparison were derived from the studies of Hankinson. ${ }^{9}$ Analyses were repeated for two exposure metrics calculated at the time of each test, cumulative RCF exposure (fibre-months/ cc) and duration of RCF employment in a production job task (production years). A cubic spline function of age with four knots was found to be significantly predictive of FVC and $\mathrm{FEV}_{1}$ $(p<0.001)$, as determined by a generalised additive model. Collinearity was examined among time-varying variables including age, cumulative cigarette pack-years of smoking, cumulative fibre exposure and production years. Correlations were 0.12 for pack-years-cumulative fibre, 0.28 for production years-cumulative pack-years, 0.37 for age-cumulative fibre, 0.47 for age-cumulative pack-years and 0.50 for age-production years. The minimisation of age collinearities was addressed by modelling age as a non-linear spline function. Cumulative fibre and production years were modelled categorically.

The longitudinal analysis utilised a two-stage mixed effects linear regression model. The first stage modelled the trajectory of each subject's measurements over time as a restricted cubic spline function of age $\left(\mathrm{a}_{\mathrm{ij}}\right){ }^{6}$ Turning points of the spline function (knots) were determined by visual inspection and best fit. Changing the number of knots, for example, from four to three, did not improve the model. The first stage model included the RCF exposure metric $\left(c_{i j}\right)$ expressed categorically, and other timedependent covariates $\left(\mathrm{z}_{\mathrm{ij}}\right)$, including cumulative pack-years, current smoking at time of test, and per cent weight change since last test. A random subject-specific average effect was included in the stage one model. This effect was modelled at the second stage as a function of subject-specific covariates $z_{i}$ and a population average intercept (Int). Fixed covariates included plant site and subject weight at initial test. These two models were combined to yield a linear mixed-effects model of the form:

$$
Y_{i j}=I n t+\beta_{1}^{\prime} a_{i j}+\beta_{2}^{\prime} c_{i j}+\beta_{3}^{\prime} a_{i j} * c_{i j}+\gamma_{1}^{\prime} z_{i j}+\gamma_{2}^{\prime} z_{i}+e_{i j}+b_{i}
$$

where $e_{i j}$ and $b_{i}$ are random effects for time and subject, respectively, assumed to be normally distributed with zero means. These two sources of variation were combined and modelled together assuming equal correlation between paired observations. An additional component of variation was modelled by including technician. The FVC and $\mathrm{FEV}_{1}$ measurements of 14 different technicians (over 17 years) were analysed as a random effect and significantly improved model fit, based on the likelihood ratio test statistic. Intraclass correlation coefficients that measure the per cent variability among measurements by the same technician, divided by total variability, were obtained. These were $1.3 \%$ and $0.7 \%$ for $\mathrm{FVC}$ and $\mathrm{FEV}_{1}$, respectively.

The parameter (vector) of primary interest described the exposure-specific effects of ageing on yearly decline in lung function. Analyses were repeated for two exposure metrics calculated at the time of each test, cumulative RCF exposure (fibre-months/cc) and duration of RCF production employment (production years). For the analysis of current male workers $(n=933)$, the three categories of cumulative RCF exposure were $\leq 15$ (50th percentile), $>15-60$ (80th percentile) and $>60$ fibremonths/cc. These categories were chosen since they provided significant differences between each pair of mean cumulative exposure values. These groups also provided a sufficient number 
Table 1 Descriptive statistics for 933 currently employed males, 244 currently employed females, and 219 formerly employed males at last test date

\begin{tabular}{|c|c|c|c|c|}
\hline & \multicolumn{4}{|c|}{ Cumulative exposure (fibre-months/cc) at last test } \\
\hline & $\leq 15$ & $>15-60$ & $>60$ & All \\
\hline \multicolumn{5}{|l|}{ Currently employed males $(n=933)$} \\
\hline Number (\%) & $470(50.4)$ & $270(28.9)$ & $193(20.7)$ & 933 \\
\hline Mean weight $(\mathrm{kg})$ & 89.6 & 92.8 & 90.0 & 90.6 \\
\hline Mean cumulative pack-years (smokers only) & 15.8 & 19.7 & 23.8 & 19.1 \\
\hline Current smoking status: number (\%) & $96(20.4)$ & $69(25.6)$ & $69(35.8)$ & \\
\hline Mean number of spirometry tests & 3.4 & 5.5 & 6.0 & 4.5 \\
\hline Mean FVC as a per cent of predicted & 98.6 & 96.7 & 94.7 & 97.3 \\
\hline Mean $\mathrm{FEV}_{1}$ as a per cent of predicted & 95.6 & 92.6 & 90.2 & 93.6 \\
\hline & \multicolumn{4}{|c|}{ Cumulative exposure (fibre-months/cc) at last test } \\
\hline & \multicolumn{2}{|c|}{$\leq 15$} & $>15$ & All \\
\hline Mean cumulative pack-years ( $\mathrm{n}=119$, smokers only) & \multicolumn{2}{|c|}{16.7} & 19.5 & 17.3 \\
\hline Current smoking status: number (\%) & \multicolumn{2}{|c|}{$56(29.3)$} & $20(37.8)$ & \\
\hline Mean production years at last test ( $n=144$; excludes non-production workers) & \multicolumn{2}{|c|}{4.3} & 12.8 & 7.0 \\
\hline Mean number of spirometry tests & \multicolumn{2}{|c|}{3.8} & 5.8 & 4.2 \\
\hline Mean FVC as a per cent of predicted & \multicolumn{2}{|c|}{99.6} & 93.7 & 98.3 \\
\hline \multirow[t]{3}{*}{ Mean $\mathrm{FEV}_{1}$ as a per cent of predicted } & \multicolumn{2}{|c|}{97.4} & 90.8 & 95.9 \\
\hline & \multicolumn{4}{|c|}{ Cumulative exposure (fibre-months/cc) at last test } \\
\hline & $\leq 15$ & $>15-60$ & $>60$ & All \\
\hline \multicolumn{5}{|l|}{ Formerly employed males $(n=219)$} \\
\hline Number (\%) & $114(52.1)$ & $63(28.8)$ & $42(19.2)$ & 219 \\
\hline Mean age (years) & 52.7 & 54.9 & 62.4 & 55.2 \\
\hline
\end{tabular}

of subjects and tests in each exposure category and allowed comparison with our previous study. ${ }^{6}$ Duration of production employment included four production year categories: (1) non (zero)-production workers (13th percentile), (2) $\leq 7$ production years (with the total of the first two categories at the 50th percentile), (3) >7-15 production years (80th percentile) and (4) $>15$ production years. The latter were similar to the last two percentiles of cumulative exposure categories. The lowest cumulative exposure category ( $\leq 15$ fibre-months/cc) represents less than 3 years of cumulative fibre exposure at current recommended guidelines of 0.5 fibres/cc. Exposure categories were collapsed for analysis of females.

For currently employed males, age slopes were calculated at midpoints of intervals at ages $30,40,50$, and at 60 years for those older than 55. This method of reporting effects within four age groups was necessary to adequately adjust for the nonlinear and accelerated loss in lung function with ageing. Because lung development may increase in young adults, estimates of slope are not shown for ages less than 25 or for cells with less than five subjects. Subjects with multiple tests contributed to estimates of the trends over time.

For the cross-sectional analyses, spirometric data at both first and last test for current males were analysed. These analyses included the same restricted spline function and main effects as the longitudinal model except for weight change. Technician was modelled as a random effect. Analyses were performed using the procedure PROC MIXED, SAS for Windows, Version 9.2.

\section{RESULTS}

Descriptive statistics for the 933 currently employed males, 244 currently employed females and 219 formerly employed males are provided in table 1 by cumulative RCF exposure category at the time of their last test date. Fibre exposure is categorised into one of three cumulative RCF exposure levels representing $\leq 15$, $>15-60$, and $>60$ fibre-months/cc. There were 193 currently employed males $(20.7 \%)$ with a cumulative RCF exposure $>60$ fibre-months/cc. The proportion of females and former workers in the highest exposure category was similar at $21.2 \%$ and $19.2 \%$, respectively. Mean age and number of years in production increased with increasing exposure category. Mean per cent of predicted lung function (FVC and $\mathrm{FEV}_{1}$ ) decreased with increasing exposure category for all three study populations. While predicted values account for age, height, sex and race differences, the mean cumulative number of pack-years smoked for current males and females also increased with increasing cumulative RCF fibre exposure. Males with higher cumulative RCF exposure were more likely to be a current smoker (35.8\%) than those in the lowest exposure group 
(20.4\%). Thus, while per cent of predicted lung function showed a decrease with increasing exposure for all groups, this approach was unadjusted for other time-related factors that affect changes in lung function, such as cumulative pack-years and weight gain.

\section{Cross-sectional analysis at first PFT}

First test results from cross-sectional analyses of 933 currently employed males are shown in table 2. Average exposure effects on FVC and $\mathrm{FEV}_{1}$ were calculated for two levels of cumulative RCF exposure, compared to the reference group with the lowest level of exposure ( $\leq 15$ fibre-months/cc). For 933 males, the effect of exposure on FVC and $\mathrm{FEV}_{1}$ for subjects in the highest exposure category ( $>60$ fibre-months/cc) compared to the reference group was significant $(\mathrm{p}<0.01)$ at -198.2 and $-154.8 \mathrm{ml}$, respectively. This cross-sectional analysis also shows the expected change with respect to pack-years of smoking, being a current smoker and body weight. Cross-sectional analyses were also conducted using the last test date on $716(77 \%)$ subjects who provided more than one PFT. Results were similar.

\section{Longitudinal change by cumulative fibre-months/cc}

Longitudinal changes in FVC and $\mathrm{FEV}_{1}$ for currently employed males with at least one PFT are summarised in figure 1 for three cumulative exposure categories across four age groups. The regression model included age, categorical cumulative RCF exposure, plant, initial weight, per cent change in weight, cumulative pack-years and smoking status (yes/no) at time of test. The FVC and $\mathrm{FEV}_{1}$ measurements of 14 different technicians were analysed as a random effect and significantly improved model fit, based on the likelihood ratio test statistic. Intraclass correlation coefficients were $1.3 \%$ and $0.7 \%$ for FVC and $\mathrm{FEV}_{1}$, respectively. Measurements for FVC and $\mathrm{FEV}_{1}$ were adjusted for height, race and spirometer. Values represent the change (slope) at each age group per exposure level. Significance was determined by $95 \%$ CIs. If intervals did not overlap, mean rates of change were judged to be statistically significant at the $5 \%$ level. While statistical significance was observed for FVC between some cells with respect to cumulative RCF exposure (eg, $\leq 15$ vs $>60$ fibre-months/cc at age 40), there was no consistent pattern demonstrating increasing loss in FVC with increasing exposure category. For FVC, initial weight and weight gain were statistically significant $(p<0.001)$. Similar findings apply to $\mathrm{FEV}_{1}$ with the additional significant finding of cumulative pack-years and current smoking status. Separate analyses (data not shown) were conducted for subjects providing two or more tests and for cumulative RCF exposure beginning at time of study initiation (1987). Trends remained essentially unchanged for both analyses, although the increased CIs resulting from smaller numbers reduced significant findings among some cells. An interaction effect of current smoking and exposure was also explored using several models including estimates calculated for each age category and exposure level (data not shown). No interaction effect was observed.

Figure 2 illustrates both the cross-sectional and longitudinal changes in $\mathrm{FEV}_{1}$ beginning at age 40 for the cohort of 933 currently employed males. The predicted $\mathrm{FEV}_{1}$ adjusted to age 40 for each worker was obtained from the cross-sectional model across all cumulative RCF exposure categories. Cross-sectionally there is a residual $-83.7 \mathrm{ml}$ loss in $\mathrm{FEV}_{1}$ at age 40 in the 15-60 fibre-months/cc exposure category compared to the reference group. The longitudinal data demonstrate subsequent yearly losses such as $-33 \mathrm{ml}$ at age $40,-43 \mathrm{ml}$ at age 50 , and $-45 \mathrm{ml}$ at age 60 (figure 1). No persistent exposure-related accelerated decline in $\mathrm{FEV}_{1}$ existed either as related to historical exposures prior to first test or ongoing exposures from 1987 to 1994. The slope of the line representing the highest exposure category does not decline at a rate faster than the lower exposure category for either $\mathrm{FEV}_{1}$ (figure 2) or FVC (data not shown).

\section{Longitudinal change by production years}

Workers were also classified by category of production years at the time of each test. Levels of lung function in production categories were compared to the non-production category. The change in FVC and $\mathrm{FEV}_{1}$ expressed in $\mathrm{ml}$ per year was determined across the same age groups. Similar to the findings with cumulative RCF exposure, there was no consistent pattern of increasing longitudinal loss in lung function (data not shown). Within each exposure category the decrease from age 30 to 60 was comparable. Initial weight and weight gain were statistically significant. Cumulative pack-years and current smoking status were additional significant contributing factors to $\mathrm{FEV}_{1}$ decline. The coefficients for initial weight, per cent weight change and smoking were nearly identical to those reported with cumulative fibre exposure.

\section{Female and former male workers}

The 244 currently employed female workers and 219 male former workers who left employment prior to 1987 were also evaluated. All had at least one PFT with no history of asthma. The longitudinal results mirror those of the larger cohort of currently exposed male workers. When analysing females for the two oldest age categories (age 40 and 50), no significant exposure-related decline was observed (data not shown). For example, at age 40 there was a 31 and $28 \mathrm{ml}$ decline in $\mathrm{FEV}_{1}$ at $\leq 15$ and $>15$ fibre-months/cc, respectively. At age 50 there was a 33 and $32 \mathrm{ml}$ decline in $\mathrm{FEV}_{1}$ at $\leq 15$ and $>15$ fibre-months/cc,

Table 2 Cross-sectional analysis $\dagger$ of FVC and $\mathrm{FEV}_{1}$ at first test date for 933 currently employed males compared to the lowest exposed group $\ddagger$

\begin{tabular}{|c|c|c|}
\hline Subjects at first test $(n=933)$ & FVC & $\mathrm{FEV}_{1}$ \\
\hline Slope at age $40(95 \% \mathrm{CI}) \S$ & $-35.7^{* * *}(-42.7$ to -28.6$)(\mathrm{ml} /$ year) & $-37.1^{* *}(-42.9$ to -31.2$)(\mathrm{ml} /$ year) \\
\hline Cumulative fibre (15-60 fibre-months/cc) $\ddagger$ & $-50.7 \mathrm{ml}$ & $-83.7 \mathrm{ml}$ \\
\hline Cumulative fibre (>60 fibre-months/cc) $\ddagger$ & $-198.2 \mathrm{ml}^{* *}$ & $-154.8 \mathrm{ml}^{* *}$ \\
\hline Current smoker at time of test & $-121.2 \mathrm{ml}^{*}$ & $-193.3 \mathrm{ml}^{* * *}$ \\
\hline Weight & $-4.1 \mathrm{ml} / \mathrm{kg}^{* *}$ & $-3.8 \mathrm{ml} / \mathrm{kg}^{* * *}$ \\
\hline Categorical plant (4 df) & $p=0.47$ & $p=0.26$ \\
\hline
\end{tabular}

${ }^{*} \mathrm{p}<0.05 ; * \mathrm{p}<0.01 ; * * \mathrm{p}<0.001$.

tRegression model included restricted cubic spline function of age, categorical cumulative fibre (fibre-months/cc), continuously modelled pack-years (pack-year), current smoker at time of test (yes/no), continuously modelled body weight $(\mathrm{kg})$, dummy variables describing five plant locations. Spirometer measurements were adjusted for height, race and spirometer. Technician was modelled as a random effect.

‡Compared to reference group having $<15$ RCF fibre-months/cc exposure.

$\S$ Mean age at first test was 36.5 years. 
Figure 1 Adjusted longitudinal change $\left(\mathrm{ml} /\right.$ year) for FVC (A) and $\mathrm{FEV}_{1}(\mathrm{~B})$ by cumulative fibre exposure (f-mo/cc) for current male employees without asthma. Also shown is the number of tests $(n)$ contributing to each age and exposure category (see text for model). (A) FVC: Initial weight $(-4.2 \mathrm{ml} / \mathrm{kg})$ and per cent weight change $(-9.4 \mathrm{ml} / \%)$ significant at $p<0.001$. (B) $F E V_{1}$ : Cumulative pack-years $(-6.9 \mathrm{ml} / \mathrm{pk}-\mathrm{yr})$, current smoking $(-42.1 \mathrm{ml})$, initial weight $(-3.1 \mathrm{ml} / \mathrm{kg})$ and per cent weight change $(-9.1 \mathrm{ml} / \%)$ are significant at $\mathrm{p}<0.01$.
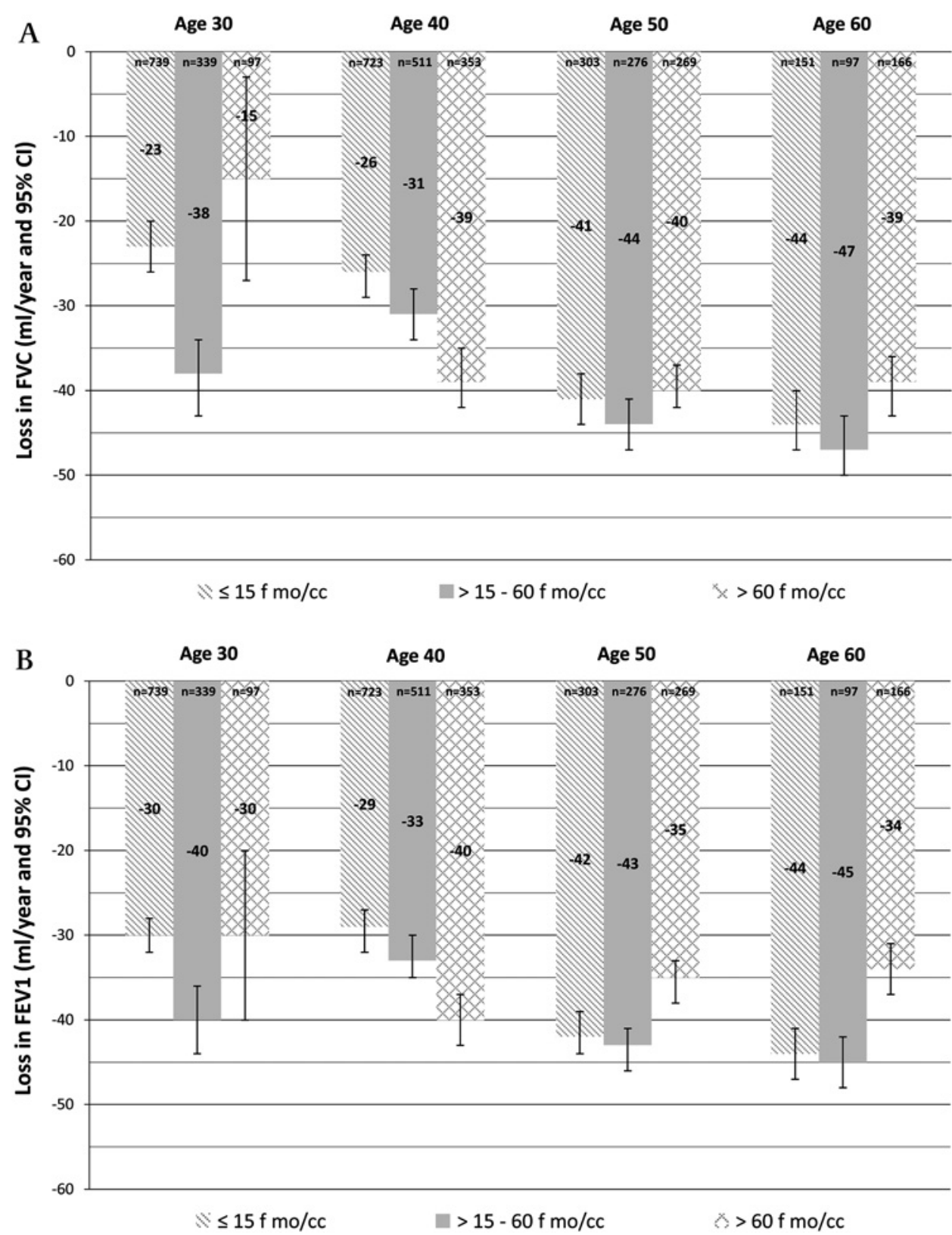

respectively. As before, statistically significant declines in $\mathrm{FEV}_{1}$ were observed with current smoking status, cumulative packyears smoked and per cent weight change.

For former male workers, the model also included the variable 'time since last employment' to evaluate decline or improvement after leaving the workplace; however, no significant difference was observed (data not shown). The mean time since last RCF exposure was 16.5 years (table 1 ). At the two oldest age categories (age 50 and 60), no significant exposure-related decline was observed. At age 50 there was a 47, 41 and $43 \mathrm{ml}$ decline in $\mathrm{FEV}_{1}$ at $\leq 15,>15-60$ and $>60$ fibre-months/cc, respectively. At age 60 , there was a 49,42 and $44 \mathrm{ml}$ decline in $\mathrm{FEV}_{1}$ at $\leq 15$, $>15-60$ and $>60$ fibre-months/cc, respectively. The model did show statistically significant declines in $\mathrm{FEV}_{1}$ with current smoking status, cumulative pack-years smoked and per cent weight change.

\section{DISCUSSION}

Important study strengths are the 17 years of follow-up, 5243 measurements of lung function, inclusion of females and former workers, and extensive ongoing and historical RCF exposure estimates. Because cumulative exposure is often highly correlated with other time-dependent variables, a statistical method was used to control for the potential confounding effects of age. This issue is important, as changes in FVC and $\mathrm{FEV}_{1}$ are nonlinear and decline faster with advancing age. The cross-sectional analysis (table 2) demonstrates a significant loss in FVC $(-198.2 \mathrm{ml})$ and $\mathrm{FEV}_{1}(-154.8 \mathrm{ml})$ in the highest cumulative RCF exposure category prior to initial test, which is consistent with earlier findings. ${ }^{6}$ Cross-sectional analyses do not adequately address time-dependent changes that correlate with age such as pack-years and weight gain ${ }^{10}$; as these are positively correlated, it is difficult to estimate the effect of each independently.

When 933 currently employed males were each assigned a level of cumulative RCF exposure and modelled longitudinally by age groups, no consistent exposure-related decline in FVC or $\mathrm{FEV}_{1}$ was found (figure 1). Our longitudinal model has the advantage of partitioning the non-linear effects of ageing, and it smoothes age effects across all intervals and increases the precision of the estimates shown in smaller slope CIs. This 


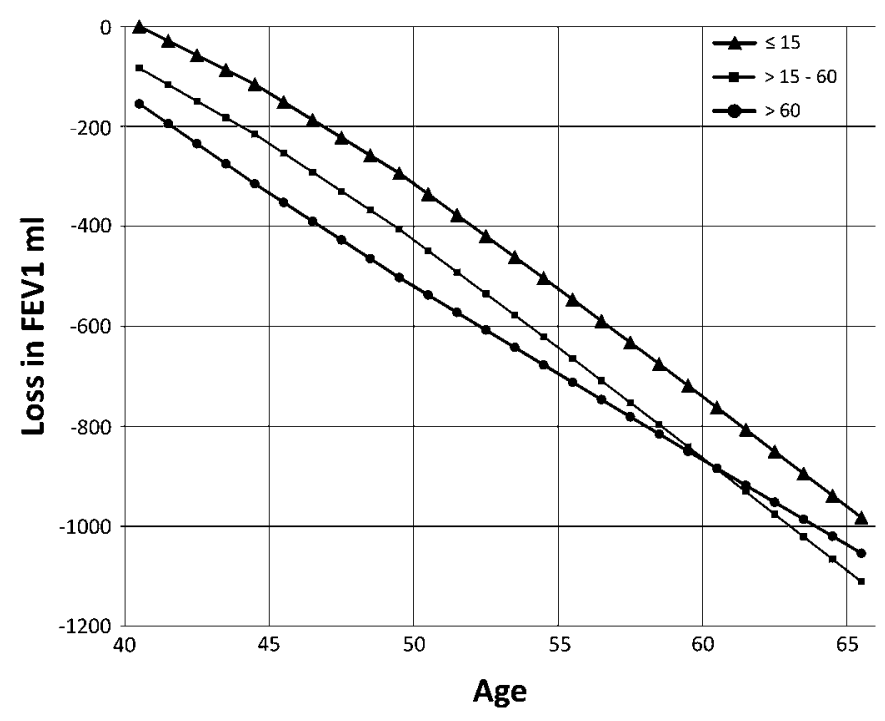

Figure 2 Longitudinal change in $\mathrm{FEV}_{1}(\mathrm{ml})$ among 933 currently exposed males by cumulative fibre exposure (f-mo/cc). The combined effects on $\mathrm{FEV}_{1}$ from our longitudinal and cross-sectional models are shown beginning at age 40 for the largest cohort of 933 currently employed males. For example, at age 40, the intercept identifies $\mathrm{a}-83.7 \mathrm{ml}$ loss in FEV 1 for workers in the 15-60 $\mathrm{f} \mathrm{mo/cc}$ exposure category. This information is derived from the cross-sectional data from table 2. The longitudinal data demonstrate subsequent yearly losses such as $-33 \mathrm{ml}$ at age $40,-43 \mathrm{ml}$ at age 50 , and $-45 \mathrm{ml}$ at age 60 (data from figure 1). Since the slope of the line representing the highest exposure category does not decline at a rate faster than the lower exposure category, no persistent exposure-related accelerated decline either as related to historical exposure or on-going exposure from 1987 to 2004 is observed.

increased precision improved study power to detect change as demonstrated with the significant findings related to current smoking status, pack-years of smoking, initial weight and weight gain. Longitudinal analyses were also conducted using three knots for the spline function at ages 25, 35 and 45, and again at 25, 35 and 55 . When three were used, all slopes were attenuated and the differences at age 40 became non-significant. We felt it was important to model changes in slope where most of the data were observed (age 35-45) and to allow slopes to differ between ages 45 and 55 and for those older than 55 , since this group had the largest proportion of highly exposed subjects. Other longitudinal models were explored (only persons contributing more than one PFT, elimination of the first PFT, etc). In all cases, trends remained similar, although the decreased number of tests and wider CIs reduced the ability to detect significant changes. Consequently, our findings were reported for the model with the greatest precision.

Differences between cross-sectional and longitudinal estimates of exposure-response in studies with time-dependent variables, as observed herein, have been reported by others. ${ }^{11}$ In subject-specific longitudinal analyses of normally distributed responses such as FVC and $\mathrm{FEV}_{1}$, slopes may be obtained by first estimating subject-specific rates of change, then averaging across all subjects. We feel that this approach is stronger than population-based cross-sectional studies, which model mean rates of change from all subjects grouped by age. For example, Hendrick modelled factors known to influence lung function and manipulated the data experimentally so that cross-sectional and longitudinal contributions could be assessed. ${ }^{11}$ A change in the mean response may include differences between subjects due to other sources than that which is attributed to the covariate.
Thus, cross-sectional studies analysis may be more susceptible to population heterogeneity.

There is evidence for a possible healthy worker effect as the decline in pulmonary function in the highest exposure group converges with the lowest (figure 2). This finding is also seen with the highest exposure category at ages 50 and 60 where there is significantly less decline in $\mathrm{FEV}_{1}$ (figure 1). An approach taken to investigate a potential healthy worker effect was to evaluate former workers who are traditionally considered to have higher historical exposures and may have left due to potential health problems. ${ }^{6}$ Overall, when compared to current males, former workers tended to have slightly larger declines in FVC at ages 50 and 60 years. This trend is not, however, as consistent with $\mathrm{FEV}_{1}$. The greater lung function decline in FVC and in some circumstances $\mathrm{FEV}_{1}$ could also be associated with higher exposure to RCF and dust during the earlier manufacturing years or by unknown exposures after leaving RCF employment. Similar to current workers, former workers did not show increasing loss in lung function with higher cumulative fibre exposure. Our study design incorporated a comparison group of low cumulative fibre exposure and nonproduction workers as an internal reference group, rather than comparison to a general population which may have employed and unemployed individuals.

In the present study, analyses of decline in FVC and $\mathrm{FEV}_{1}$ were conducted at several ages to control for the non-linear and accelerated loss in function with ageing as well as changes that occur in other risk factors over time. We observed the smallest decreases in FVC among the youngest age group but at the highest exposure category (figure 1). This group, however, had a lower mean body weight $(82.1 \mathrm{~kg})$ compared to the low and intermediate exposure categories (to 86.6 and $90.2 \mathrm{~kg}$ ). This suggests that the lowest loss in lung function occurs among the lightest subjects and that weight is highly associated with lung function changes. Because smoking and weight are confounders, in addition to the potential for participation bias related to smoking, the convergence of the line for the highest and lowest exposure categories cannot be completely explained by a healthy worker effect. While a healthy worker effect is a possible explanation for the non-significant trends, it is unlikely to be primary.

Participation bias is always a concern. Our participation rate for current males and females with initially normal spirometry was $93.3 \%$ (3438 tests/3685 opportunities) and for those having an initial FVC or $\mathrm{FEV}_{1} / \mathrm{FVC} \%$ less than the lower limit of normal, the rate was only slightly lower at 90.4\% (630 tests/697 opportunities). Those with lower lung function, however, were also twice as likely to be current smokers. Smoking also may be a factor that explains differences in results from this study and others. Studies investigating changes in lung function among RCF exposed workers have been conducted in Europe ${ }^{512}$ as well as those previously reported by our team in the USA. ${ }^{6}{ }^{13}$ In a European cross-sectional study of currently exposed RCF workers, small but significant declines in $\mathrm{FEV}_{1}$ were found between increasing cumulative exposure (fibre-ml/years) and smoking. ${ }^{5}$ A follow-up cross-sectional analysis reported similar findings with $\mathrm{FEV}_{1}$ and FVC in current male smokers. ${ }^{12}$ In the USA, cross-sectional declines among RCF workers with 10 years of production employment were reported among male smokers for FVC and $\mathrm{FEV}_{1}{ }^{13}$ In the current longitudinal study no interaction between smoking and exposure was observed. These differences may be due to smoking habits. In the Cowie ${ }^{12}$ and LeMasters $^{13}$ studies, 38\% and 41\% were current smokers, respectively, versus $25 \%$ in the current study. While it is 
generally true that longitudinal declines are frequently considered to be more reliable than cross-sectional analyses, these are susceptible to errors caused by selective participation in multiple surveys, learning effects, healthy survivor and healthy worker effects, and instrument or technician changes. ${ }^{14} 15$

Recently, a longitudinal (1990-2006) historical summary of RCF exposure for workers in manufacturing plants and at customer facilities was reported. ${ }^{16}$ This study included four of our five manufacturing locations, plus several others. In general, the annual average time-weighted average (TWA) concentrations decreased with the greatest change in the early 1990s. Exposures at our study sites have been well characterised. ${ }^{7}$ The majority of job title exposure estimates have remained relatively stable for the plant sites, even though changes were made with respect to workplace ventilation. The most significant improvements, however, have been directed towards those jobs with highest exposure. Of the 122 job titles active in 2001, Rice reported $79 \%$ were at less than or equal to $0.25 \mathrm{f} / \mathrm{cc}, 14 \%$ ranged from $>0.25$ to $0.5 \mathrm{f} / \mathrm{cc}$ and $7 \%$ exceeded $0.5 \mathrm{f} / \mathrm{cc}$. Industry guidelines recommend an 8-h TWA exposure of $0.5 \mathrm{f} / \mathrm{ccc}^{17}$ It is important to recognise, however, that the number of workers within a specific job title can change and a decrease in exposure does not always decrease the percentage of job titles within an exposure category.

In conclusion, no consistent longitudinal decline in FVC or $\mathrm{FEV}_{1}$ with increasing RCF exposure category was observed, although cross-sectional changes were observed for subjects in the highest exposure category. Critical to this analysis was the recognition that lung function declines with age are non-linear and accelerate in older age groups who also have the longest duration of exposure. To our knowledge, this specific analysis strategy has not been published for evaluating longitudinal change in lung function among adults, although a similar methodology has been used in children. ${ }^{18}$ Longitudinal change in lung function has been investigated by others; however, these studies frequently use linear regression or GEE methods of analysis that may not adequately adjust for age-related accelerated declines. ${ }^{19-23}$ The methods used for this analysis are recommended when undertaking longitudinal analyses of lung function.

Acknowledgements We thank the men and women who participated in this longitudinal study as well as other personnel at the plant sites who assisted the study team. The authors are deeply grateful to numerous staff members and scientific consultants who over the years helped us design, implement, analyse, and interpret these data.

Funding The Refractory Ceramic Fiber Coalition (2300 N Street NW, Room 6178, Washington, DC USA 20037) provided funding for this study.

Competing interests James E Lockey has received grants from the Refractory Ceramic Fiber coalition through the University of Cincinnati College of Medicine for directing and analysing the data of the medical surveillance program and for salary support of contributing faculty and support staff. Eric K Borton, Carol H Rice, Linda Levin and Timothy Hilbert have received research funding from the Refractory Ceramic Fiber Coalition for participation in the medical surveillance program. Grace $\mathrm{K}$ LeMasters has received funding as a research investigator for the Refractory Ceramic Fiber Coalition and for the presentation of preliminary results of the medical surveillance program and for salary support. Roy T McKay has received faculty salary support from the Refractory Ceramic Fiber Coalition for his role in the design and analysis of this study.
Patient consent Obtained.

Ethics approval This study was conducted with the approval of the University of Cincinnati Institutional Review Board.

Provenance and peer review Not commissioned; externally peer reviewed.

\section{REFERENCES}

1. Bunn WB, Bender JR, Hesterberg TW, et al. Recent studies of man-made vitreous fibers. J Occup Med 1993:35:101-13.

2. Mast RW, McConnell EE, Anderson R, et al. Studies on the chronic toxicity (inhalation) of four types of refractory ceramic fibers in male Fischer 344 rats. Inhal Toxicol 1995; 7:425-67.

3. Mast RW, McConnell EE, Hesterberg TW, et al. Multiple-dose chronic inhalation toxicity study of size-separated kaolin refractory ceramic fiber in male Fischer rats. Inhal Toxicol 1995:7:469-502.

4. McConnell EE, Mast RW, Hesterberg TW, et al. Chronic inhalation toxicity of a kaolin-based refractory ceramic fiber in Syrian golden hamsters. Inhal Toxicol 1995; 7:503-32.

5. Trethowan MN, Burge PS, Rossiter CE, et al. Study on the respiratory health of workers in seven European plants that manufacture ceramic fibres. Occup Environ Med 1995;52:97-104.

6. Lockey JE, Levin LS, Lemasters GK, et al. Longitudinal estimates of pulmonary function in refractory ceramic fiber manufacturing workers. Am J Respir Crit Care Med 1998;157:1226-33.

7. Rice $\mathbf{C H}$, Lockey JE, Lemasters G, et al. Estimation of historical and current employee exposure to refractory ceramic fibers during manufacturing and related operations. Appl Occup Environ Hyg 1997;12:54-61.

8. Rice CH, Levin LS, Borton EK, et al. Exposure to refractory ceramic fibers in manufacturing and related operations: a 10 year update. J Occup Environ Hyg 2005:2:462-73.

9. Hankinson JL, Odencrantz JR, Fedan KB. Spirometric reference values from a sample of the general U.S. Population. Am J Respir Crit Care Med 1999:159:179-87.

10. McKay RT, Lockey JE, Lemasters G, et al. Weight gain and lung function: considerations for surveillance studies. J Occup Environ Med 1999;41:596-604.

11. Hendrick DJ, Becklake M, Hanley JA. Discordance between cross-sectional and longitudinal studies for the effect of dust on COPD: why? COPD 2005;2:395-404

12. Cowie HA, Wild P, Beck J et al. An epidemiological study of the respiratory health of workers in the European refractory ceramic fibre industry. Occup Environ Med 2001;58:800-10.

13. Lemasters G, Lockey J, Levin L, et al. An industry-wide pulmonary study of men and women manufacturing refractory ceramic fibers. Am J Epidemiol 1998; 148:910-19

14. Chinn S, Jarvis D, Svanes $C$, et al. Sources of variation in forced expiratory volume in one second and forced vital capacity. Eur Respir J 2006;27:767-73.

15. Hnizdo $\mathbf{E}, \mathrm{Yu}$ L, Freyder $\mathrm{L}$, et al. The precision of longitudinal lung function measurements: monitoring and interpretation. Occup Environ Med 2005;62:695-701.

16. Maxim LD, Allshouse J, Fairfax RE, et al. Workplace monitoring of occupational exposure to refractory ceramic fiber-a 17 year retrospective. Inhal Toxicol 2008;20:289-309.

17. Anon. Refractory Ceramic Fibers Coalition and Occupational Safety and Health Administration Voluntary Worker Protection Program Renewed. http://www.rcfc.net/ psphtw.htm (accessed 1 Apr 2010).

18. Berhane K, Gauderman WJ, Stram D0, et al. Statistical issues in studies of the long-term effects of air pollution: the southern California children's health study. Stat Sci 2004:19:414-49.

19. Attfield MD, Hodous TK. Pulmonary function of U.S. coal miners related to dust exposure estimates. Am Rev Respir Dis 1992;145:605-9.

20. Naidoo RN, Robins TG, Seixas N, et al. Differential respirable dust related lung function effects between current and former South African coal miners. Int Arch Occup Environ Health 2005:78:293-302.

21. Mastrangelo G, Zanibellato R, Fedeli U, et al. Exposure to hydrogen peroxide at TLV level does not induce lung function changes: A longitudinal study. Int $J$ Environ Health Res 2005:15:313-17.

22. Gainet $\mathbf{M}$, Thaon I, Westeel V, et al. Twelve-year longitudinal study of respiratory status in dairy farmers. Eur Respir J 2007;30:1383-9.

23. Wang $\mathbf{X}$, Zhang $\mathrm{H}$, Sun $\mathrm{B}$, et al Cross-shift airway responses and long-term decline in FEV1 in cotton textile workers. Am J Crit Care Med 2008;177: $316-20$ 